\title{
Efecto del tiempo y temperatura de almacenamiento en los parámetros físico-químicos y de color de frutos de aguaymanto (Physalis peruviana)
}

\section{Effect of storage time and temperature on the physico-chemical and color parameters of aguaymanto (Physalis peruviana) fruits}

\author{
Frank F. Velásquez-Barreto ${ }^{1,2, a}$, Daniel A. Rafael-Delgado ${ }^{1,2, b,}{ }^{,}$, Edson E. Ramírez-Tixe ${ }^{1,2, c}$ \\ ${ }^{1}$ Proyecto de investigación docente, Vicepresidencia de investigación, Universidad Nacional Autónoma de Chota, Chota, \\ Perú. \\ ${ }^{2}$ Escuela Profesional de Ingeniería Agroindustrial, Facultad de Ciencias Agrarias, Universidad Nacional Autónoma de \\ Chota, Chota, Perú. \\ a Ph.D., 作velasquez@unach.edu.pe, (1D https://orcid.org/0000-0001-8954-9769 \\ b Bach., 囚 danielrafd@gmail.com, (iD https://orcid.org/0000-0002-5639-3734 \\ cMg., 囚 \\ * Autor de Correspondencia: Tel. +51921607495 \\ http://dx.doi.org/10.25127/riagrop.20221.782
}

http://revistas.untrm.edu.pe/index.php/RIAGROP revista.riagrop@untrm.edu.pe

Recepción: 20 de noviembre 2021

Aprobación: 15 de diciembre 2021

Este trabajo tiene licencia de Creative Commons. Attribution-NonCommercial-ShareAlike $\quad 4.0$ International Public License - CC-BY-NC-SA 4.0

\section{Resumen}

Los frutos de aguaymanto (Physalis peruviana) son comercializados en fresco, en diversas regiones de Sudamérica y del mundo. No obstante, existen algunos problemas relacionados a la pérdida de calidad, debido al mal manejo del almacenamiento postcosecha. Por ello, este estudio evaluó el efecto del tiempo y temperatura de almacenamiento postcosecha, sobre las propiedades fisicoquímicas e índice de madurez de frutos de aguaymanto. Se utilizó un diseño factorial de dos factores (temperatura y tiempo de almacenamiento). Los frutos de aguaymanto fueron colocados en empaques de polietileno tereftalato perforados y fueron almacenados en oscuridad a $4{ }^{\circ} \mathrm{C}, 12{ }^{\circ} \mathrm{C}$ y $20^{\circ} \mathrm{C}$. Las mediciones de Brix, $\mathrm{pH}$, acidez, índice de madurez y parámetros de color $\left(\mathrm{L}^{*}, \mathrm{a}^{*} . \mathrm{b}^{*}\right)$ se realizaron a los 0, 8, 17 y 27 días. Los frutos de aguaymanto que fueron almacenados a bajas temperaturas $\left(4{ }^{\circ} \mathrm{C}\right)$ mostraron una ligera variación en su índice de madurez y presentaron una reducción en los parámetros $L^{*}$ y b $b^{*}$ un 
incremento del parámetro a* a los 7 días de almacenamiento. Se observó un efecto significativo $(\mathrm{p}<0.05)$ del tiempo y temperatura de almacenamiento postcosecha sobre el Brix, $\mathrm{pH}$, acidez, índice de madurez y los parámetros de color de frutos de aguaymanto.

Palabras claves: Aguaymanto, propiedades fisicoquímicas, color, índice de madurez.

\begin{abstract}
The fruits of goldenberry (Physalis peruviana) are marketed fresh in various regions of South America and the world; however, there are some problems related to the loss of quality due to poor postharvest storage management. Therefore, this study evaluated the effect of postharvest storage time and temperature on goldenberry fruits' physicochemical properties and maturity index. For that purpose, a two-factor factorial design (temperature and storage time) was used. Goldenberry fruits were placed in perforated polyethylene terephthalate packages and were stored in the dark at $4{ }^{\circ} \mathrm{C}, 12^{\circ} \mathrm{C}$, and $20^{\circ} \mathrm{C}$. The measurements of Brix, $\mathrm{pH}$, acidity, maturity index and color parameters $\left(\mathrm{L}^{*}, \mathrm{a}^{*} . \mathrm{b}^{*}\right)$ were carried out at $0,8,17$, and 27 days. Goldenberry fruits stored at low temperatures $\left(4^{\circ} \mathrm{C}\right)$ showed a slight variation in their maturity index, and they presented a reduction in the $\mathrm{L}^{*}$ and $\mathrm{b}^{*}$ parameters and an increase in the $\mathrm{a}^{*}$ parameter after 7 days of storage. A significant effect $(p<0.05)$ of postharvest storage time and temperature on Brix, $\mathrm{pH}$, acidity, maturity index, and color parameters of goldenberry fruits was observed.
\end{abstract}

Keywords: Goldenberry, Physicochemical properties, color, maturity index.

\section{INTRODUCCIÓN}

Los alimentos frescos con buenas características físicas, sensoriales y químicas son de importancia para el actual mercado, porque los consumidores buscan productos que, con buena calidad, cuiden su salud (Li et al., 2017). Dentro de los alimentos frescos, se aprecia a las frutas, las cuales contienen vitaminas, minerales $\mathrm{y}$ compuestos bioactivos. Los frutos nativos como aguaymanto (Physalis peruviana), cocona (Solanum sessiliflorum), sauco (Sambucus nigra L.), guinda (Prunus cerasus) pitahaya amarilla (Selenicereus megalanthus) y sanky (Corryocactus brevistylus) son una alternativa para la comercialización de frutos frescos (Obregón-La Rosa et al., 2021). Los frutos de P. peruviana L. son de color amarillo, redondos, con cierto brillo característico debido a la presencia de cera. Algunos nombres que reciben los frutos de $P$. peruviana son aguaymanto (Perú), uchuva (Colombia) y uvilla (Ecuador). El mayor componente de estos frutos es el agua (81.33\%) y presentan un Brix de 13.73-14.30 y una acidez de 1.9 a $2.1 \%$ (Puente et al., 2011). Así mismo, se ha reportado un contenido de vitamina $C$ de 24.21 a 46 mg/100 g de pulpa (Ramadan et al.; 2013; Málaga et al., 2013; Velásquez-Barreto, 2018) y la presencia de ciertos compuestos con potencial bioactivo como los fisalinas, fitoesteroles, y winalónidos (Puente et al., 2011).

Durante el almacenamiento postcosecha de frutos frescos, ciertas características físicas y sensoriales pueden alterarse debido a cambios fisiológicos propios de cada especie. También, algunos compuestos secundarios como compuestos fenólicos, antocianinas, carotenoides, entre otros, pueden incrementar o reducir su concentración debido a los procesos 
de síntesis y degradación (Dziedzic et al., 2020). Es muy importante determinar la composición de metabolitos secundarios, sobre todo si cumplen una función en beneficio del organismo, además de conocer el tiempo óptimo en cual se puede consumir un producto fresco sin perder un contenido significativo de estos compuestos (Aglar et al., 2017). No obstante, los frutos frescos son consumidos por sus cualidades físicas y sensoriales que, en combinación con los índices de madurez, son buenos indicadores a la hora de determinar la calidad óptima de un fruto fresco (BalagueraLópez et al., 2016).

Diversos trabajos han demostrado que el tiempo de vida útil de los frutos frescos depende del tipo de almacenamiento al que son sometidos. Frutos almacenados en condiciones ambientales presentan un corto tiempo de vida útil, debido a que las reacciones de síntesis y degradación son intensas, más aún si el almacenamiento se realiza a altas temperaturas, si el fruto es climatérico, presencia de etileno, presencia de agentes patógenos (hongos y levaduras), etc. Por ello, es importante aplicar alguna tecnología de almacenamiento postcosecha como la refrigeración, atmósferas modificadas o atmósferas controladas, ya sea en forma individual o en combinación (Piccolo et al., 2020). Se ha reportado que los frutos de $P$. peruviana son climatéricos, presentan una alta tasa de respiración $\mathrm{y}$, por ello, su actividad metabólica es alta. Estas características de los frutos pueden ocasionar la pérdida o cambios de las características físicas, sensoriales y de metabolitos secundarios de interés (Pinzón et al., 2015). Por ello, la aplicación de tecnologías de manejo postcosecha es muy importante a fin de conservar las características e incrementar el tiempo de vida útil de los frutos de $P$. peruviana.
Por otro lado, resulta de vital importancia determinar el efecto de las principales variables o factores que afectan el almacenamiento postcosecha, porque estos pueden afectar la calidad del fruto. Algunos frutos son sensibles a bajas temperaturas de almacenamiento y estos pueden presentar ciertas zonas con tonalidad oscura debido al daño por frío (Bonat et al., 2016). Así mismo, el contenido de los compuestos bioactivos puede verse alterado (aumento o reducción) según la temperatura de almacenamiento. De la misma manera, las características físicas y sensoriales pueden sufrir estos cambios (Brizzolara et al., 2020). Por ello, es importante conocer la temperatura óptima de almacenamiento. Otros factores que pueden afectar el tiempo de almacenamiento son la humedad relativa, tipo de envase, etc.

Los frutos de P. peruviana son comercializados y consumidos en fresco en diversas regiones de Sudamérica y del mundo. No obstante, existen algunos problemas relacionados a la pérdida de calidad debido a un inadecuado manejo del almacenamiento postcosecha (Barirega et al., 2014). Diversos factores pueden alterar tanto la calidad física, sensorial o química y se puede afectar el tiempo de vida útil. Así mismo, como los frutos de P. peruviana presentan un corto período de vida útil y se ha reportado un alto contenido de compuestos bioactivos con posibles beneficios para salud, el objetivo de este trabajo fue evaluar el efecto del tiempo y temperatura de almacenamiento postcosecha sobre las propiedades fisicoquímicas e índice de madurez de frutos de aguaymanto (Physalis peruviana). 


\section{MATERIALES Y MÉTODOS}

\subsection{Lugar de estudio}

La labor de investigación se efectuó en las instalaciones del laboratorio de Análisis y Control de Calidad de Productos Agroindustriales de la Escuela Profesional de Ingeniería Agroindustrial, Facultad de Ciencias Agrarias de la Universidad Nacional Autónoma de Chota, Chota - Perú.

\subsection{Materia prima}

Se recolectaron frutos de aguaymanto $(P$. peruviana L.) de la comunidad Conga el Verde, distrito de Chalamarca, Chota (2388 m s. n. m.). Los frutos fueron sanos (sin daños mecánicos o atacados por insectos), de tamaño homogéneo con pesos promedio de $3.3 \mathrm{~g}$ sin cáliz, en un nivel de madurez de 3, según Norma Técnica Colombiana, NTC 4580, Instituto Colombiano de Normas Técnicas y Certificación (ICONTEC, 1999). Para cada tratamiento se utilizó tres muestras de 150 g cada una para cada temperatura de almacenamiento.

\subsection{Diseño experimental}

Se utilizó un diseño factorial de dos factores, que fueron la temperatura $\left(4^{\circ} \mathrm{C}, 12{ }^{\circ} \mathrm{C}\right.$ y $\left.20^{\circ} \mathrm{C}\right)$ y tiempo de almacenamiento $(0,8,17$ y 27 días $)$. Se colocaron $300 \mathrm{~g}$ de frutos de aguaymanto en empaques de polietileno tereftalato perforados. Luego, fueron almacenados en oscuridad a la temperatura de $4{ }^{\circ} \mathrm{C}, 12^{\circ} \mathrm{C}$ y $20^{\circ} \mathrm{C}$. Además, se ejecutaron las mediciones de las propiedades físico-químicas y de los parámetros de color a $\operatorname{los} 0,8,17$ y 27 días. Finalmente, se realizaron tres réplicas para cada tratamiento.

\subsection{Propiedades fisicoquímicas}

El contenido de sólidos solubles (ํㅜix) de los frutos fue realizado por medición directa mediante un refractómetro manual (ISOLAB) a
$20{ }^{\circ} \mathrm{C}$ (AOAC, 2003). La determinación del pH de los frutos se realizó por medición directa del zumo previamente extraído utilizando un $\mathrm{pH}$ metro (HANNA Instruments) y se siguió el procedimiento descrito por AOAC (2003). La acidez titulable de los frutos se determinó y expresó como porcentaje de ácido málico (AOAC, 2003).

\section{5. Índice de madurez}

El índice de madurez se determinó mediante la relación entre los sólidos solubles (Brix) y la acidez titulable (Norma Técnica Colombiana, NTC 4580, Instituto Colombiano de Normas Técnicas y Certificación - ICONTEC, 1999).

\subsection{Parámetros de color}

Los parámetros de color ( $\mathrm{L}^{*}$-Luminosidad, $\mathrm{a}^{*}$ rojo-verde, $b^{*}$-amarillo-azul) de los frutos de aguaymanto se determinaron con la medición directa de la superfie de los frutos y se tuvo como refencia la zona ecuatorial del fruto (4 puntos opuestos) y sin presencia visual de defectos. Los parámetros CIE - $\mathrm{L}^{*}, \mathrm{a}^{*}, \mathrm{~b}^{*}$ se determinaron con el empleo de un colorímetro (PCE Instruments, Spectrophotometer, modelo: PCE - CSM 8), calibrado previamente. Las condiciones de la medición fueron: apertura de diámetro $8 \mathrm{~mm}$, plato blanco de referencia, iluminación estándar D65 y observador a $10^{\circ}$ (CIE, 2004).

\subsection{Análisis estadístico}

Los datos obtenidos en las variables de respuesta (sólidos solubles totales, $\mathrm{pH}$, acidez, índice de madurez y parámetros de color) fueron analizados con un diseño factorial de dos factores con $95 \%$ de significancia $(\alpha=0.05)$ y se realizaron las pruebas de comparación de medias de Tukey. Todas las pruebas estadísticas 
se realizaron con el software estadístico RStudio.

\section{RESULTADOS Y DISCUSIÓN}

\subsection{Brix}

Durante el proceso de maduración, los frutos degradan almidón, pectina y otros polímeros para producir energía mediante la respiración, como consecuencia se genera un incremento en el contenido de sólidos solubles (Hernández y Arjona, 2001). Los frutos de aguaymanto almacenados a $4{ }^{\circ} \mathrm{C}, 12{ }^{\circ} \mathrm{C}$ y $20^{\circ} \mathrm{C}$ presentaron un Brix de 10.60 a 13.13; de 10.60 a 15.44 y de 10.60 a 16.20 , respectivamente (tabla 1). En general, los frutos de aguaymanto que se almacenaron a bajas temperaturas $\left(4^{\circ} \mathrm{C}\right.$ y $\left.12{ }^{\circ} \mathrm{C}\right)$ presentaron un menor Brix durante el tiempo de almacenamiento. A los 17 días de almacenamiento, se observó un incremento en el Brix de frutos de aguaymanto almacenados a $12{ }^{\circ} \mathrm{C}$, en comparación con los frutos almacenados a $4{ }^{\circ} \mathrm{C}$. Esto demuestra que la temperatura de almacenamiento de $4{ }^{\circ} \mathrm{C}$ reduce la producción de sólidos solubles (Brix) durante el periodo de almacenamiento. Estos resultados concuerdan con los obtenidos por Ramírez et al. (2005), quienes mencionan que bajas temperaturas reducen la concentración de sólidos solubles durante el almacenamiento postcosecha, debido a que, bajo esta condición, el ritmo de respiración del fruto se reduce. Por otro lado, se observó un efecto significativo del tiempo y temperatura de almacenamiento sobre el Brix de los frutos de aguaymanto $(p<0.05)$.

Tabla 1. Influencia del tiempo y la temperatura de almacenamiento postcosecha sobre el Brix, pH y acidez de frutos de aguaymanto

\begin{tabular}{|c|c|c|c|c|c|c|}
\hline \multicolumn{7}{|c|}{ Temperatura $\left({ }^{\circ} \mathrm{C}\right)$} \\
\hline Día & 4 & 12 & 20 & 4 & 12 & 20 \\
\hline \multicolumn{4}{|c|}{${ }^{\circ}$ Brix } & \multicolumn{3}{|c|}{$\mathrm{pH}$} \\
\hline 0 & $10.60 \pm 0.41 \mathrm{a}, \mathrm{A}$ & $10.60 \pm 0.4 \mathrm{a}, \mathrm{A}$ & $10.60 \pm 0.4 \mathrm{a}, \mathrm{A}$ & $3.50 \pm 0.02 \mathrm{a}, \mathrm{A}$ & $3.50 \pm 0.02 \mathrm{a}, \mathrm{A}$ & $3.50 \pm 0.02 \mathrm{a}, \mathrm{A}$ \\
\hline 8 & $10.80 \pm 0.18 \mathrm{a}, \mathrm{A}$ & $10.95 \pm 0.13 a, A$ & $11.29 \pm 0.06 b, B$ & $3.53 \pm 0.03 a, A$ & $3.25 \pm 0.04 b, B$ & $3.38 \pm 0.07 c, C$ \\
\hline 17 & $12.50 \pm 0.14 \mathrm{a}, \mathrm{B}$ & $12.60 \pm 0.39 a, B$ & $13.15 \pm 0.13 b, C$ & $3.39 \pm 0.05 a, B$ & $3.54 \pm 0.06 \mathrm{ab}, \mathrm{A}$ & $3.61 \pm 0.03 b, C$ \\
\hline 27 & $13.13 \pm 0.88 \mathrm{a}, \mathrm{B}$ & $15.44 \pm 0.42 b, C$ & $16.20 \pm 0.53 b, D$ & $3.38 \pm 0.02 a, B$ & $3.44 \pm 0.03 \mathrm{~b}, \mathrm{C}$ & $3.67 \pm 0.02 c, D$ \\
\hline \multicolumn{7}{|c|}{ Acidez } \\
\hline 0 & $3.50 \pm 0.02 \mathrm{a}, \mathrm{A}$ & $3.50 \pm 0.02 \mathrm{a}, \mathrm{A}$ & $3.50 \pm 0.02 \mathrm{a}, \mathrm{A}$ & & & \\
\hline 8 & $3.07 \pm 0.06 a, B$ & $3.18 \pm 0.04 b, B$ & $2.80 \pm 0.06 c, B$ & & & \\
\hline 17 & $3.15 \pm 0.02 \mathrm{a}, \mathrm{C}$ & $3.06 \pm 0.03 b, C$ & $3.07 \pm 0.06 \mathrm{ab}, \mathrm{C}$ & & & \\
\hline 27 & $3.74 \pm 0.04 a, D$ & $3.07 \pm 0.04 b, C$ & $2.68 \pm 0.03 c, D$ & & & \\
\hline
\end{tabular}

Nota: Diferentes letras minúsculas indican diferencia significativa $(\mathrm{p}<0.05)$ entre el Brix, $\mathrm{pH}$ y acidez para el tiempo de almacenamiento. Diferentes letras mayúsculas indican diferencia significativa $(\mathrm{p}<0.05)$ entre el Brix, $\mathrm{pH}$ y acidez para la temperatura de almacenamiento. 


\section{2. $\mathrm{pH}$ y acidez}

El pH y la acidez son parámetros que indican los cambios fisiológicos ocurridos en los frutos durante el almacenamiento postcosecha. Estos parámetros son inversamente proporcionales (Bernal et al., 1998). Los frutos de aguaymanto almacenados a $20{ }^{\circ} \mathrm{C}$ presentaron un mayor $\mathrm{pH}$ y menor acidez que los frutos de aguaymanto almacenados a $4{ }^{\circ} \mathrm{C}$ y $12{ }^{\circ} \mathrm{C}$ (tabla 1). El comportamiento del $\mathrm{pH}$ y acidez a las temperaturas de $4{ }^{\circ} \mathrm{C}$ y $12{ }^{\circ} \mathrm{C}$ fue variable durante el periodo de almacenamiento (tabla 1 ), debido a un posible efecto de la temperatura sobre la fisiología de los frutos de aguaymanto. Los ácidos orgánicos de los frutos responsables del $\mathrm{pH}$ y la acidez de los frutos son productos del metabolismo de los polímeros presentes y la concentración de estos ácidos puede alterarse por un factor externo como la temperatura de almacenamiento (Martínez-González et al., 2017). Así mismo, se encontró un efecto significativo del tiempo y temperatura de almacenamiento $(p<0.05)$ en el $\mathrm{pH}$ y la acidez de los frutos de aguaymanto.

\section{3. Índice de madurez}

El índice de madurez de los frutos de aguaymanto es un parámetro que permite conocer la calidad de los frutos de aguaymanto para consumo en fresco o para procesamiento y este se obtiene de la relación entre el Brix y la acidez del fruto (Norma Técnica Colombiana
NTC 4580, ICONTEC, 1999). Los frutos de aguaymanto presentaron un índice de madurez de 3 a $4.5 \mathrm{y}$, de acuerdo a la norma Norma Técnica Colombiana NTC 4580 (ICONTEC, 1999), este se puede clasificar en un fruto con tipo de color 3 y 4 (fruto de color anaranjado claro con o sin zonas verdes), que indica una madurez adecuada. El índice de madurez de los frutos de aguaymanto se incrementó en las tres temperaturas de almacenamiento $\left(4^{\circ} \mathrm{C}, 12{ }^{\circ} \mathrm{C}, \mathrm{y}\right.$ $20{ }^{\circ} \mathrm{C}$ ) durante el tiempo de almacenamiento. No obstante, este incremento del índice de madurez fue menor en los frutos almacenados a $4{ }^{\circ} \mathrm{C}$ (figura 1). A partir del día 17, el incremento del índice de madurez en los frutos fue mayor a las temperaturas de $12 \quad{ }^{\circ} \mathrm{C}$ y $20 \quad{ }^{\circ} \mathrm{C}$ en comparación con los frutos almacenados a $4{ }^{\circ} \mathrm{C}$ (figura 1). Esto indica que los frutos almacenados a $4{ }^{\circ} \mathrm{C}$ reducen su actividad metabólica y esto se observa con la menor producción de sólidos solubles y acidez en comparación con las otras temperaturas de almacenamiento (tabla 1). Se han observado ligeros incrementos del índice de madurez durante el almacenamiento postcosecha a bajas temperaturas en otros frutos y es atribuido a la formación de azúcares y la degradación de ácidos orgánicos (Lanchero et al., 2007; OlivaresTenorio et al., 2017; Peña et al., 2015; Peña et al., 2013; Osorio y Roldán, 2003). Se encontró un efecto significativo del tiempo y temperatura de almacenamiento $(p<0.05)$ en el índice de madurez de los frutos de aguaymanto. 


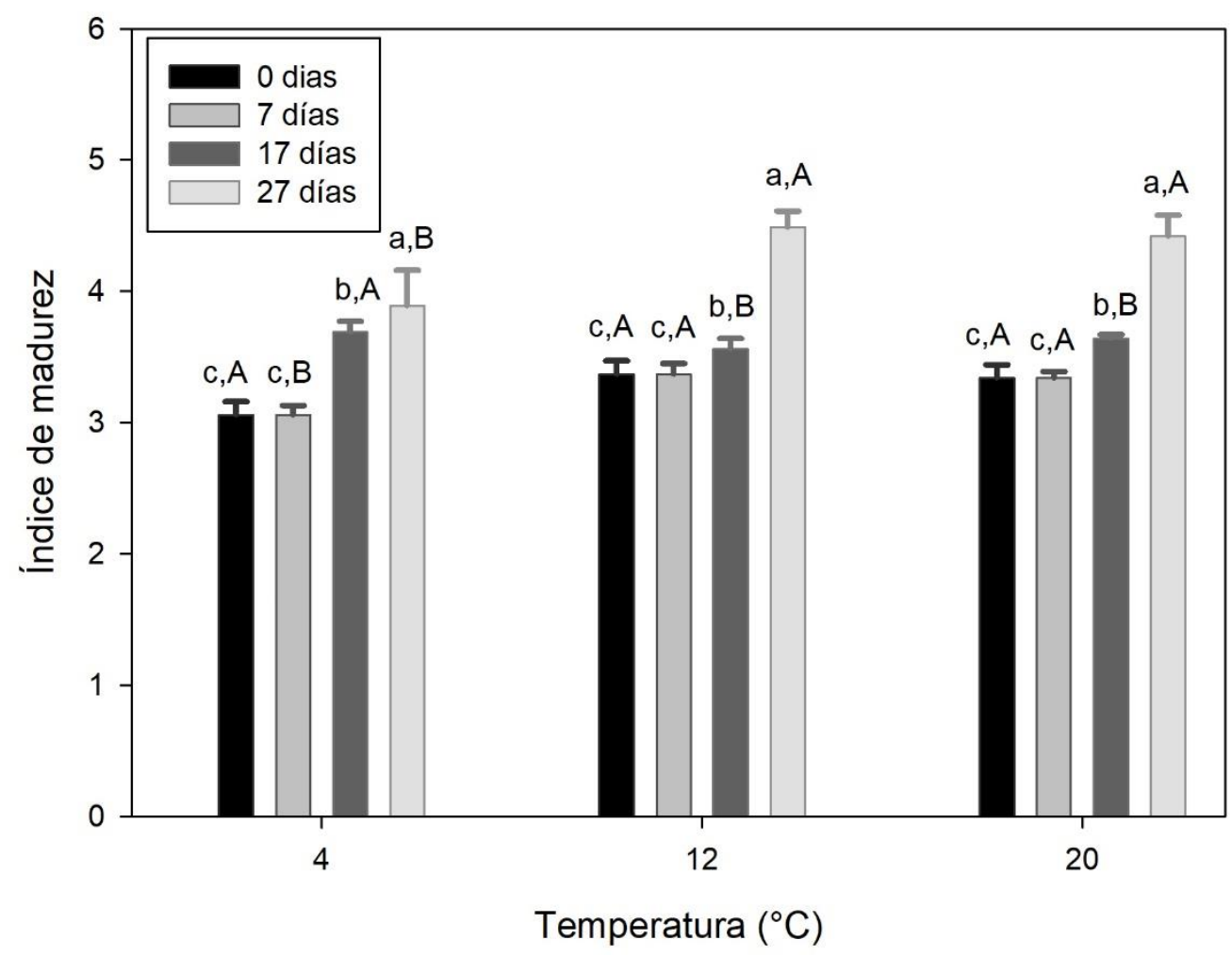

Figura 1. Influencia del tiempo y temperatura de almacenamiento postcosecha sobre el índice de madurez de frutos de aguaymanto. Diferentes letras minúsculas y mayúsculas indican diferencia significativa $(\mathrm{p}<0.05)$ entre el índice de madurez para tiempo de almacenamiento y para la temperatura de almacenamiento, respectivamente.

\subsection{Parámetros color}

El parámetro de color $\mathrm{L}^{*}$, a los 7 días de almacenamiento se redujo a las tres temperaturas de almacenamiento y esta reducción fue mayor a la temperatura de $4{ }^{\circ} \mathrm{C}$. Esto indica que los frutos almacenados a más bajas temperaturas pueden sufrir una mayor reducción en su actividad metabólica. Después de los 7 días de almacenamiento, el comportamiento del parámetro $L^{*}$ fue variable y se observó mayores valores de este parámetro a temperaturas de almacenamiento más bajas (tabla 2). Comportamientos similares fueron observados por Olivares-Tenorio et al. (2017) y Novoa et al. (2006) para frutos de aguaymanto almacenados a temperaturas de $4{ }^{\circ} \mathrm{C}$ y $12{ }^{\circ} \mathrm{C}$ y este comportamiento fue atribuido a la disminución de la tasa de respiración y ausencia de etileno (Pinzón et al., 2015).

El parámetro de color $\mathrm{a}^{*}$, a los 7 días de almacenamiento, se incrementó a las tres temperaturas de almacenamiento $\mathrm{y}$ este incremento fue mayor a la temperatura de $4{ }^{\circ} \mathrm{C}$ (tabla 2). Esta situación puede indicar que los frutos almacenados presentaron una mayor acumulación de metabolitos secundarios como los carotenoides, que son los responsables del típico color anaranjado del fruto (MartínezGonzález et al., 2017). Después de los 7 días de almacenamiento, el comportamiento del parámetro $\mathrm{a}^{*}$ fue variable y se observó menores valores de este parámetro a $4{ }^{\circ} \mathrm{C}$. Esto indicaría 
una posible reducción del contenido de carotenoides en los frutos, debido a una posible estabilización a la baja temperatura de almacenamiento. Según Camacho et al. (2013) y Pinzón et al. (2015), los frutos almacenados a temperaturas a bajas muestran una etapa inicial de estabilización que se relaciona con la etapa de estrés donde se desarrollan cambios bioquímicos en el metabolismo fotosintético y la alteración del proceso fisiológico, debido a disminución de la tasa de respiración.

En relación al parámetro de color $\mathrm{b}^{*}$, a los 7 días de almacenamiento, este parámetro se redujo a $4{ }^{\circ} \mathrm{C}$ y $12{ }^{\circ} \mathrm{C}$ y se incrementó a $20{ }^{\circ} \mathrm{C}$. Después de los 7 días de almacenamiento, el valor de este parámetro se incrementó a las tres temperaturas de almacenamiento (tabla 2). Estas variaciones, a los 7 días de almacenamiento en el parámetro $\mathrm{b}^{*}$, también fueron observadas en el parámetro $L^{*}$ y $a^{*}$ a bajas temperaturas de almacenamiento, que indicaría que este tiempo corresponde a un periodo de estabilización de los frutos a bajas temperaturas de almacenamiento. Durante el proceso de maduración, los frutos cambian de color verde a amarillo. Por ello, el parámetro b*, también es un indicativo del avance del proceso de maduración (Balaguera-Lopez et al., 2016), porque los mayores valores de este parámetro fueron observados conforme el tiempo de almacenamiento se incrementó a las tres temperaturas de almacenamiento.

Se encontró un efecto significativo del tiempo y temperatura de almacenamiento $(p<0.05)$ en los parámetros de color $L^{*}, a^{*}$ y $b^{*}$ de los frutos de aguaymanto. Efectos similares fueron observados con el índice de madurez (figura 1) y esto indica que la variación de parámetros de color $\left(\mathrm{L}^{*}, \mathrm{a}^{*} \mathrm{y} \mathrm{b}^{*}\right)$ durante el almacenamiento postcosecha a diferentes temperaturas que podrían relacionarse con el índice de madurez (Oliveira et al., 2016).

Tabla 2. Influencia del tiempo y temperatura de almacenamiento postcosecha sobre los parámetros de color $\left(L^{*}, a^{*}\right.$ y $\left.b^{*}\right)$ de los frutos de aguaymanto

\begin{tabular}{|c|c|c|c|c|c|c|c|c|c|}
\hline & \multicolumn{9}{|c|}{ Temperatura $\left({ }^{\circ} \mathrm{C}\right)$} \\
\hline \multirow{2}{*}{ Día } & 4 & 12 & 20 & 4 & 12 & 20 & 4 & 12 & 20 \\
\hline & & $\mathbf{L}^{*}$ & & & $\mathbf{a}^{*}$ & & & $\mathbf{b}^{*}$ & \\
\hline 0 & $58.85 \pm 3.64 \mathrm{a}, \mathrm{A}$ & $58.85 \pm 3.64 a, A$ & $58.85 \pm 3.64 a, A$ & $7.31 \pm 0.89 \mathrm{a}, \mathrm{A}$ & $7.31 \pm 0.89 \mathrm{a}, \mathrm{A}$ & $7.31 \pm 0.89 \mathrm{a}, \mathrm{A}$ & $50,69 \pm 2,24 a, A$ & $50.69 \pm 2.24 a, A$ & $50.69 \pm 2.24 \mathrm{a}, \mathrm{A}$ \\
\hline 8 & $39.58 \pm 1.37 \mathrm{~b}, \mathrm{~B}$ & $40.96 \pm 2.56 b, B$ & $53.65 \pm 3.53 \mathrm{ab}, \mathrm{A}$ & $42.12+2.17 \mathrm{a}, \mathrm{B}$ & $24.98 \pm 6.40 \mathrm{~b}, \mathrm{~B}$ & $19.09 \pm 0.82 b, B$ & $36,10 \pm 3,94 a, B$ & $30.91 \pm 2.04 a, B$ & $54.18 \pm 3.16 \mathrm{~b}, \mathrm{~A}$ \\
\hline 17 & $57.75 \pm 1.67 \mathrm{a}, \mathrm{A}$ & $56.16 \pm 1.92 \mathrm{a}, \mathrm{A}$ & $52.52 \pm 1.84 \mathrm{~b}, \mathrm{~B}$ & $12.14 \pm 1.33 a, C$ & $17.11 \pm 0.74 b, C$ & $14 \pm 0.94 a, C$ & $52,16 \pm 3,49 a, A$ & $50.39 \pm 2.48 \mathrm{a}, \mathrm{A}$ & $55.26 \pm 3.07 \mathrm{a}, \mathrm{A}$ \\
\hline 27 & $59.57 \pm 2.03 a, A$ & $57.39 \pm 3.08 \mathrm{a}, \mathrm{A}$ & $54.92 \pm 3.96 \mathrm{ab}, \mathrm{A}$ & $9.42 \pm 1.02 \mathrm{a}, \mathrm{A}$ & $21.53 \pm 0.88 b, B$ & $20.13 \pm 1.03 b, B$ & $52,29 \pm 3,59 a, A$ & $55.56 \pm 1.96 \mathrm{a}, \mathrm{A}$ & $57.22 \pm 5.42 \mathrm{a}, \mathrm{A}$ \\
\hline
\end{tabular}

Nota: Diferentes letras minúsculas indican diferencia significativa $(\mathrm{p}<0.05)$ entre los parámetros de color para el tiempo de almacenamiento. Diferentes letras mayúsculas indican diferencia significativa $(\mathrm{p}<0.05)$ entre los parámetros de color para la temperatura de almacenamiento. 


\section{CONCLUSIONES}

Se observó un efecto significativo $(p<0.05)$ del tiempo y temperatura de almacenamiento postcosecha sobre el Brix, $\mathrm{pH}$, acidez, índice de madurez y los parámetros de color: $L^{*}, a^{*}, b^{*}$ de frutos de aguaymanto (P. peruviana). Los frutos de aguaymanto que fueron almacenados a bajas temperaturas $\left(4{ }^{\circ} \mathrm{C}\right)$ mostraron una ligera variación en su índice de madurez. No obstante, se observó un incremento de este índice a partir de los 7 días de almacenamiento. Los frutos almacenados a bajas temperaturas $\left(4^{\circ} \mathrm{C}\right.$ y $\left.12{ }^{\circ} \mathrm{C}\right)$ presentaron una reducción en los parámetros $\mathrm{L}^{*}$ y $b^{*}$ y un incremento del parámetro $a^{*}$ a los 7 días de almacenamiento y luego el comportamiento fue inverso, que indica un posible efecto de la temperatura de almacenamiento en la fisiología del fruto. Se podrían realizar futuros estudios para esclarecer el efecto de la temperatura sobre los compuestos bioactivos de frutos de aguaymanto durante un tiempo de almacenamiento prolongado.

\section{Referencias}

Aglar, E., Ozturk, B., Guler, S.K., Karakaya, O., Uzun, S. \& Saracoglu, O. (2017). Effect of modified atmosphere packaging and 'Parka' treatments on fruit quality characteristics of sweet cherry fruits (Prunus avium L. '0900 Ziraat') during cold storage and shelf life. Scientia Horticulturae, 222, 162-168. https://doi.org/10.1016/j.scienta.2017.05.024

AOAC. (2003). Métodos de análisis de la asociación oficial de química analítica para determinar humedad, fibra, cenizas, grasas y proteínas. Recuperado de http://www.fao.org/3/b-ap802s.pdf

Balaguera-López, H.E., Martínez-Cárdenas, C.A. \& Herrera-Arévalo, A. (2016). Behavior of cape gooseberry (Physalis peruviana L.). Bioagro, 28(2), 117124.

Barirega, A. (2014). Potential for Value Chain Improvement and Commercialization of Cape Gooseberry (Physalis peruviana L.) for Livelihood
Improvement in Uganda. Ethnobotany Research and Applications, 12, 131-140.

Bernal, I; Ramírez, B. \& Academia Colombiana de Ciencias Exactas, Físicas y Naturales. (1998). Análisis de alimentos. Santafé de Bogotá.

Bonat, C.G., Ghanem, A. \& Su-Ling, M. (2016). Influence of freezing process and frozen storage on the quality of fruits and fruit products. Food Reviews International, 32(3), 280-304. https://doi.org/10.1080/87559129.2015.1075212

Brizzolara, S., Manganaris, G.A., Fotopoulos, V., Watkins, C.B. \& Tonutti, P. (2020). Primary Metabolism in Fresh Fruits During Storage. In Frontiers in Plant Science, $11, \quad$ p. 80. https://www.frontiersin.org/article/10.3389/fpls.2020 .00080

Camacho, J., Cerquera, N. \& Gutiérrez, N. (2013). Determinación del color del exocarpio como indicador de desarrollo fisiológico y madurez en la guayaba pera (Psidium guajava cv. Guayaba pera), development and maturity in pear guava (Psidium guajava cv. Pear). Revista EIA, 10(19), 79-89. http://www.scielo.org.co/pdf/eia/n19/n19a07.pdf

CIE. (2004). CIE 15. Technical report: Colorimetry. 3rdedition. http://cie.co.at/publications/colorimetry-3rd-edition

Dziedzic, E., Błaszczyk, J., Bieniasz, M., Dziadek, K. \& Kopeć, A. (2020). Effect of modified (MAP) and controlled atmosphere (CA) storage on the quality and bioactive compounds of blue honeysuckle fruits (Lonicera caerulea L.). Scientia Horticulturae, 265, 109226. https://doi.org/10.1016/j.scienta.2020.109226

Hernández Gómez, M. \& Arjona, H. (2001). Conservación del fruto de araza (Eugenia stipitata Mc Vaugh) durante la poscosecha mediante la aplicación de diferentes técnicas. [Tesis de Doctorado, Universidad Nacional de Colombia.]

https://repository.agrosavia.co/handle/20.500.12324/ 29736

Instituto Colombiano de Normas Técnicas y Certificación - ICONTEC. (1999). Norma Técnica Colombiana NTC (4580) - Frutas frescas. Huchuva. Especificaciones. Bogotá: ICONTEC, 15 p.

Lanchero, O., Velandia, G., Fischer, G., Varela, N.C. \& García, H. (2007). Comportamiento de la uchuva (Physalis peruviana L.) en postcosecha bajo condiciones de atmósfera modificada activa. Ciencia E Tecnología Agropecuaria, 8(1), 61-68. https://doi.org/10.21930/rcta.vol8_num1_art:84

Li, L., Zhang, M., Adhikari, B. \& Gao, Z. (2017). Recent advances in pressure modification-based 
preservation technologies applied to fresh fruits and vegetables. Food Reviews International, 33(5), 538-559. https://doi.org/10.1080/87559129.2016.1196492

Málaga, R.; Guevara, A. \& Vargas, M. (2013). Efecto del procesamiento de puré de aguaymanto (Physalis peruviana 1.), sobre los compuestos bioactivos y la capacidad antioxidante. Revista de la Sociedad Química del Perú. 79(2), 162-174.

Martínez-González, M.E., Balois Morales, R., Alia-Tejacal, I., Cortes-Cruz, M.A., Palomino-Hermosillo, Y.A., López-Gúzman, G.G., Martínez-González, M.E., Balois-Morales, R., Alia-Tejacal, I., Cortes-Cruz, M.A., Palomino-Hermosillo, Y.A. \& López-Gúzman, G.G. (2017). Postcosecha de frutos: maduración y cambios bioquímicos. Revista Mexicana de Ciencias Agrícolas, 8(19), 4075-4087. https://doi.org/10.29312/remexca.v0i19.674

Novoa, R.H., Bojacá, M., Galvis, J.A. \& Fischer, G. (2006). La madurez del fruto y el secado del cáliz influyen en el comportamiento poscosecha de la uchuva, almacenada a $12{ }^{\circ} \mathrm{C}$ (Physalis peruviana L.) *. Agronomía Colombiana, 24(1), 77-86. http://www.scielo.org.co/scielo.php?script=sci_artte xt\&pid=S0120-

99652006000100010\&lng=en\&nrm=iso\&tlng=es

Obregón-La Rosa, A.J., Augusto Elías-Peñafiel, C.C., Contreras-López, E., Arias-Arroyo, G.C. \& Bracamonte-Romero, M. (2021). Características fisicoquímicas, nutricionales y morfológicas de frutas nativas. Revista de Investigaciones Altoandinas, 23(1), 17-25. https://doi.org/10.18271/ria.2021.202

Olivares-Tenorio, M.L., Dekker, M., van Boekel, M.A. \& Verkerk, R. (2017). Evaluating the effect of storage conditions on the shelf life of cape gooseberry (Physalis peruviana L.). LWT, 80, 523-530. https://doi.org/https://doi.org/10.1016/j.lwt.2017.03.0 27

Oliveira, S.F., Gonçalves, F.J.A., Correia, P.M.R. \& Guiné, R.P.F. (2016). Physical properties of Physalis peruviana L. Open Agriculture, 1(1), 55-59. https://doi.org/doi:10.1515/opag-2016-0007

Osorio, Diaz, D.L. \& Roldan, J.C. (2003). Volvamos al campo: manual de la uchuva (No. C047. 085). Grupo Latino LTDA.

Peña, C.R.F., Cortés, M. \& Montoya, O. (2013). Evaluation of the physicochemical, physical and sensory properties of fresh cape gooseberry and vacuum impregnated with physiologically active components. Vitae Revista de La Facultad de Química Farmacéutica, 20(1), 13-22. http://www.scielo.org.co/scielo.php?script=sci_artte
xt\&pid=S0121-

$40042013000100002 \& \operatorname{lng}=$ en \&nrm=iso\&tlng=en

Peña, F., Cortés, M. \& Gil, J. (2013). Estabilidad Fisicoquímica y Funcional de Uchuva (Physalis peruviana L.) Impregnada a Vacío con Calcio y Vitaminas B9, D y E, Durante el Almacenamiento Refrigerado. Revista Facultad Nacional de Agronomía Medellín, $66 \quad$ (1), 6929-6938. http://www.scielo.org.co/scielo.php?script=sci_artte

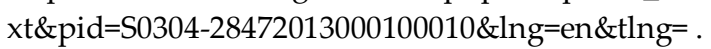

Piccolo, E.L., Martìnez Garcìa, L., Landi, M., Guidi, L., Massai, R. \& Remorini, D. (2020). Influences of Postharvest Storage and Processing Techniques on Antioxidant and Nutraceutical Properties of Rubus idaeus L.: A Mini-Review. Horticulturae, 6(4), 105. https://doi.org/10.3390/horticulturae6040105

Pinzón, E., Reyes, A., Álvarez-Herrera, J., Leguizamo, M. \& Joya, J. (2015). Postharvest behavior of cape gooseberry Physalis peruviana L. fruit under different storage temperatures. Revista de Ciencias Agrícolas, 32(2 SE-Research Article). https://doi.org/10.22267/rcia.153202.10

Pinzón, E.H., Reyes, A.J., Álvarez-Herrera, G., Leguizamo, M.F. \& Joya, J.G. (2015.) Comportamiento del fruto de uchuva Physalis peruviana L., bajo diferentes temperaturas de almacenamiento. Revista de Ciencias Agricolas, 32(2), 26 - 35.

Puente, L., Pinto-Muñoz, C., Castro, E. \& Cortés, M. (2011). Physalis peruviana Linnaeus, the multiple properties of a highly functional fruit: A review. Food Resource International, 44, 1733-1740.

Ramadan, M.F., Hassan, N.A., Elsanhoty, R.M. \& Sitohy, M.Z. (2013). Goldenberry (Physalis peruviana) juice rich in health-beneficial compounds suppresses high-cholesterol diet-induced hypercholesterolemia in rats. Journal of Food Biochemistry, 37, 708-722. doi: 10.1111/j.1745-4514.2012.00669.x.

Ramírez, J.M., Galvis, J.A. \& Fischer, G. (2005). Maduración poscosecha de la feijoa (Acca sellowiana Berg) tratada con $\mathrm{CaCl} 2$ en tres temperaturas de almacenamiento. Agronomía Colombiana, 23(1), 117127.

https://www.redalyc.org/articulo.oa?id=18031695101 5

Velásquez-Barreto, F., Riveros, A. \& Ruíz, A. (2018). El uso de encapsulantes mejora la retención de ácido ascórbico en zumo de Physalis peruviana L. atomizado: Estudio de retención y modelación del comportamiento higroscópico. Scientia Agropecuaria, 9(4), $535-542$. https://doi.org/10.17268/sci.agropecu.2018.04.09 